Original Article

\title{
Central line-associated bloodstream infections (CLABSI): Microbiology and antimicrobial resistance pattern of isolates from the Pediatric ICU of a tertiary care Indian hospital
}

\author{
Shilpa Tomar ${ }^{a}$, Rakesh Lodha ${ }^{b}$, Bimal Das ${ }^{a}$, Seema Sood ${ }^{a}$, Arti Kapil ${ }^{a, *}$ \\ a Department of Microbiology, All India Institute of Medical Sciences, New Delhi, India \\ ${ }^{\mathrm{b}}$ Department of Pediatrics, All India Institute of Medical Sciences, New Delhi, India
}

\section{A R T I C L E I N F O}

\section{Article history:}

Received 15 September 2015

Accepted 28 October 2015

Available online 27 November 2015

Keywords:

CLABSI

PICU

Microbiology

Gram-negative organisms

Antimicrobial resistance

\begin{abstract}
A B S T R A C T
Objectives: Central line-associated bloodstream infections (CLABSIs) are important and serious healthcare-associated infections (HAI), with a reported mortality of $12-25 \%$. We conducted this study to determine incidence of CLABSI in our Pediatric Intensive Care Unit (PICU), and to study the profile (gram positive or negative) of organisms causing these infections and also their antimicrobial resistance pattern.

Methods: A prospective cohort study was conducted in our PICU for a period of one and a half years from 1st August 2011 to 31st January 2013. Blood samples were collected for culture when primary or secondary bacteremia was suspected. A CLABSI was defined as per CDC guidelines. Antimicrobial susceptibility testing was done by Disk diffusion Technique using Kirby Bauer's method and interpretation was as per CLSI guidelines.

Results: There were 13 episodes of CLABSI, of which, 5 (38.5\%) were caused by Klebsiella spp., 3 (23.1\%) by Pseudomonas spp., 1(7.7\%) by Acinetobacter spp., 1 (7.7\%) by Proteus spp., 1 (7.7\%) by Enterococcus faecium, and 2 (15.3\%) by Staphylococcus epidermidis. All the Pseudomonas spp. isolates were sensitive to Ciprofloxacin whereas Acinetobacter spp. was resistant to it. Four of 5 Klebsiella spp. isolates were resistant to carbapenems and all were resistant to 3rd generation cephalosporins. Both the S. epidermidis isolates were methicillin resistant.

Conclusions: Gram-negative organisms continue to predominate HAIs in the developing countries, reflecting the suboptimal infection control. More worrisome is the rise of multidrug-resistant gram-negative bacilli, which are resistant to quinolones, 3rd and 4th generation cephalosporins, and carbapenems. Preventive and control measures are the most costeffective parameters to help contain the rising antimicrobial resistance.
\end{abstract}

(C) 2015 INDIACLEN. Published by Elsevier, a division of Reed Elsevier India, Pvt. Ltd. All rights reserved.

\footnotetext{
* Corresponding author. Tel.: +91 1126594237.

E-mail address: akapilmicro@gmail.com (A. Kapil).

http://dx.doi.org/10.1016/j.cegh.2015.10.008
}

2213-3984/C 2015 INDIACLEN. Published by Elsevier, a division of Reed Elsevier India, Pvt. Ltd. All rights reserved. 


\section{Introduction}

Intravascular catheters are indispensable for modern day healthcare. They include short-term peripheral venous and arterial catheters, short-term central venous catheters (CVCs), long-term tunneled and cuffed CVCs, and long-term peripherally inserted CVCs. ${ }^{1}$

Although such catheters provide necessary vascular access, their use puts patients at risk for local and systemic infectious complications, including local site infection, catheter-related bloodstream infection (CRBSI), septic thrombophlebitis, endocarditis, and other metastatic infections (e.g., lung abscess, brain abscess, osteomyelitis, and endophthalmitis). ${ }^{2}$

Central line-associated bloodstream infections (CLABSIs) are one of the most important HAIs associated with a high mortality rate of $12-25 \% .^{3}$ Multiple studies of the causative organisms for CRBSIs exist in the adult literature, but few such studies in the pediatric population exist. We conducted this study to determine incidence of CLABSI in our Pediatric ICU, and study the profile (gram positive or negative) of organisms causing these infections and also their antimicrobial resistance pattern.

\section{Methods}

\subsection{Study design and setting}

We conducted a prospective cohort study to measure the burden of CLABSIs in the Pediatric Intensive Care Unit (PICU) of All India Institute of Medical Sciences (AIIMS), New Delhi. We also aimed to study the profile of organisms causing these infections and their corresponding antimicrobial resistance pattern. This study was conducted in the PICU for a period of one and a half years from 1st August 2011 to 31st January 2013. Ethical clearance was taken from the Ethics Committee of AIIMS (Ref. No. IESC/T-282/01.07.2011).

\subsection{Participants}

Inclusion criteria

(a) All the children who stayed in PICU for $\geq 48 \mathrm{~h}$.

(b) Patients with no infection incubating at the time of admission to PICU, i.e. samples collected on Day 1 of the patients stay were culture negative (blood, urine, and sputum/broncho-alveolar lavage).

\section{Exclusion criteria}

(a) All the children who stayed in PICU for $<48 \mathrm{~h}$.

(b) Patients with any infection incubating at the time of admission to PICU, i.e. samples collected on Day 1 of patients stay were culture positive (blood, urine, and sputum/broncho-alveolar lavage).

\subsection{Materials and methods}

\subsubsection{CLABSI}

Case definition - This was defined as per CDC guidelines, ${ }^{3}$ i.e. a CLABSI is recovery of a pathogen from a blood culture (a single blood culture for organisms not commonly present on the skin and two or more blood cultures for organisms commonly present on the skin) in a patient who had a central line at the time of infection or within the 48-h period before development of infection. The infection cannot be related to any other infection the patient might have and must not have been present or incubating when the patient was admitted to the facility.

\subsubsection{Secondary bloodstream infection}

Bloodstream infection (BSI), which is secondary to a primary infection site, i.e. related to an infection at another site, such that the primary site of infection may have seeded the bloodstream secondarily. If the criteria for the primary site infection require a culture, then at least one organism cultured from that site must match an organism in the blood culture. ${ }^{4}$

Blood samples were collected for culture when primary or secondary bacteremia was suspected. For children $>1$ year age, 3-5 ml blood was drawn out and put in a blood culture bottle containing $50 \mathrm{ml}$ of Brain Heart Infusion (BHI) broth, whereas for infants, $1 \mathrm{ml}$ blood was put in a McCartney's bottle containing $10 \mathrm{ml}$ BHI broth.

The sample in blood culture bottle was kept in the incubator at $37^{\circ} \mathrm{C}$. After $24 \mathrm{~h}$, first subculture was done on Blood Agar (BA) and MacConkey Agar (MA). If negative, second subculture was done on BA and MA after $48 \mathrm{~h}$. After 2 weeks, final subculture was done on $\mathrm{BA}$ and $\mathrm{MA}$, before discarding the sample.

Interpretation - A single positive blood culture was considered significant for organisms not commonly present on the skin and two or more blood cultures for organisms commonly present on the skin.

\subsubsection{Antimicrobial susceptibility testing}

It was done by Disk diffusion Technique using Kirby Bauer's method and interpretation as per CLSI guidelines. ${ }^{5}$

\section{Results}

Out of 349 patients included in our study, 19 developed a BSI. All the patients who developed a BSI were on a central line during their stay in the PICU. Of these 19 patients, 13 (68.4\%) had primary BSI due to the central line, i.e. they developed central line-associated bloodstream infection (CLABSI). The remaining 6 (31.6\%) patients developed a secondary bloodstream infection, i.e. related to an infection at another site.

The incidence density of CLABSI was 5.03 per 1000 central line days calculated by the following formula:

$$
\begin{aligned}
\text { Incidence density of CLABSI } & =\frac{\text { Episodes of CLABSI }}{\text { Central line days }} \times 1000 \\
& =13 / 2580 \times 1000=5.03
\end{aligned}
$$

When calculating incidence density of CLABSIs, the Centre for Disease Control and Prevention ${ }^{6}$ (CDC) guidelines recommend that we take "central-line days" as the denominator for the calculation. 'Central line days' is calculated by daily count of patients on a central line, admitted in a healthcare facility. This adjusts risk of CLABSI with respect to the duration the central line was in place. 


\subsection{Microbiology}

The 13 episodes of CLABSI were predominantly caused by gram-negative organisms, as depicted by Table 1 .

\subsection{Antimicrobial susceptibility profile of bacterial isolates causing CLABSI}

Of the 13 bacterial isolates of CLABSI, 10 were gram-negative bacilli (GNB) and 3 were gram-positive cocci (GPC).

1) Gram-negative organisms

a) Nil-fermenters

Of the 3 Pseudomonas spp. isolates causing CLABSI, 2 were sensitive to Amikacin. None were sensitive to Imipenem. One isolate was sensitive to Meropenem. All three isolates were sensitive to Ciprofloxacin, Cefoperazone-Sulbactum, and Piperacillin-Tazobactam. The single isolate of Acinetobacter spp. was sensitive to Meropenem, Cefoperazone-Sulbactum, and PiperacillinTazobactam and resistant to Amikacin and Imipenem.

b) Enterobacteriacae family

i) Klebsiella spp. - Of the 5 Klebsiella spp. isolates, 3 were sensitive to Amikacin. Only one was sensitive to Imipenem and Meropenem. All isolates were sensitive to Ciprofloxacin and Cefoperazone-Sulbactum. Of the 5 isolates, four were sensitive to PiperacillinTazobactam.

ii) Proteus spp. - The only isolate of Proteus spp. was sensitive to all drugs except Ciprofloxacin.

2) Gram-positive organisms

i) Staphylococcus epidermidis - Both isolates of S. epidermidis were methicillin resistant. Of the two isolates, one was sensitive to Amikacin. Both isolates were sensitive to Vancomycin, Teicoplanin, and Linezolid and resistant to Erythromycin, Penicillin, and Rifampicin.

ii) Enterococcus faecium - The single isolate of E. faecium was resistant to Amikacin. It was sensitive to Gentamicin, Vancomycin, and Linezolid and resistant to Teicoplanin, Erythromycin, and Penicillin.

\section{Discussion}

The present study was undertaken to obtain information on CLABSIs in our PICU, in an attempt to have all policies and care

Table 1 - Etiology of organisms causing CLABSI in PICU.

\begin{tabular}{lc} 
Etiology & CLABSI \\
& $n=13$ \\
\hline Klebsiella spp & $5(38.5 \%)$ \\
Pseudomonas spp. & $3(23.1 \%)$ \\
Acinetobacter spp. & $1(7.7 \%)$ \\
Proteus spp. & $1(7.7 \%)$ \\
Enterococcus faecium & $1(7.7 \%)$ \\
Staphylococcus epidermidis & $2(15.3 \%)$ \\
Total & 13 \\
\hline
\end{tabular}

procedures in place. This information would also help in future development of protocols to reduce the incidence, and thus morbidity, mortality, and healthcare cost associated with CLABSI.

Multiple studies of the causative organisms for CRBSIs exist in the adult literature, but few such studies in the pediatric population exist. The National Nosocomial Infection Surveillance System survey (NNISS) of PICU patients identified coagulase-negative Staphylococcus spp. as the predominant organism associated, accounting for $37.8 \%$ of reported cases. ${ }^{7}$ The most commonly reported causative pathogens remain coagulase-negative staphylococci, Staphylococcus aureus, enterococci, and Candida spp. ${ }^{8}$ GNB accounted for $19 \%$ and $21 \%$ of CLABSIs reported to CDC and the Surveillance and Control of Pathogens of Epidemiological Importance (SCOPE) database, respectively. ${ }^{8}$

In our study, we found a predominance of GNBs causing CLABSI, in contrast to GPCs, which predominate in studies from developed countries. ${ }^{9}$ Similarly, in a previous study done in our PICU on HAIs, $96.5 \%$ of the bacterial isolates causing HAIs were GNBs, including BSIs. ${ }^{10}$ In another Indian study done in PGI, Chandigarh by Lakshmi ${ }^{11}$ et al. in the PICU, it was also found that GNBs predominated as a causative factor for nosocomial BSI. Thus, GNBs continue to dominate in developing countries where infection control practices are not optimal.

Emerging multidrug-resistant GNBs are another major challenge in the treatment of these infections. The gramnegative bacteria resistant to quinolones and all 3rd and 4th generation cephalosporins, which are important drugs for empirical treatment in ICUs, are increasing. To this, added the emergence of carabapenem resistance, it leaves very limited choice for the treatment of the patient. We also found similar trends in the CLABSI isolates of our study. Nil-fermenters are now on the rise in the healthcare settings. Colistin is the only choice left in cases of multidrug-resistant BSI isolates. Tigecycline use is not a very good treatment option in BSI patients.

Antimicrobial resistance has now become a global challenge. Extensive use of antibiotics has led to selective pressure on bacteria and emergence of multidrug-resistant strains of bacteria, thus taking us back to almost the pre-antibiotic era. ${ }^{12}$

Limitations of our study include, not looking for fungi as the causative organism of CLABSI in each and every patient. Only in high-suspicion cases, where bacterial blood cultures were negative and the patients did not respond to antibiotics, fungal blood culture was performed. Also, our study lacks any statistical conclusion.

Prevention and control of infection is one of the most important and most cost-effective parameter to contain and reduce the burden of antimicrobial selective pressure. HAIs due to multidrug-resistant organisms are a major challenge in any healthcare facility. ${ }^{12}$ For prevention of nosocomial infections, especially device-associated infections like CLABSI, education is an important and sustainable measure.

\section{Conflicts of interest}

The authors have none to declare. 


\section{R E F E R E N C E S}

1. Edgeworth J. Intravascular catheter infections. J Hosp Infect. 2009;73:323-330.

2. Morbidity and Mortality Weekly Report. Guidelines for the Prevention of Intravascular Catheter-Related Infections. vol. 51. August 9, 2002.

3. Morbidity and Mortality Weekly Report. Vital Signs: Central Line-Associated Blood Stream Infections - United States 2001 2008 and 2009. vol. 60. March 1, 2011.

4. Secondary Bloodstream Infection (BSI) Guide. CDC; June 2011.

5. CLSI. Performance Standards for Antimicrobial Susceptibility Testing; Twenty-Second Informational Supplement; 2012. M100-S22, Vol. 32, No. 3.

6. Bloodstream Infection Event (Central Line-Associated Bloodstream Infection and Non-Central Line-Associated Bloodstream Infection). CDC; January 2015.
7. Newman D. Catheter-related bloodstream infections in the pediatric intensive care unit. Semin Pediatr Infect Dis. 2006;17:20-24.

8. Wisplinghoff H, Bischoff T, Sandra M, Seifert H, Wenzel R, Edmond M. Nosocomial Bloodstream Infections in US Hospitals: Analysis of 24,179 Cases from a Prospective Nationwide Surveillance Study. CID; 2004:39.

9. Edmond B, Wallace E, McClish K, et al. Nosocomial bloodstream infections in United States hospitals: a threeyear analysis. Clin Infect Dis. 1999;29:239-244.

10. Gupta A, Kapil A, Lodha R. Burden of healthcare-associated infections in a paediatric intensive care unit of a developing country: a single centre experience using active surveillance. J Hosp Infect. 2011;78(August (4)):323-326.

11. Lakshmi S, Jayashree M, Singhi S, Ray P. Study of nosocomial primary bloodstream infections in a pediatric intensive care unit. J Trop Pediatr. 2006;53(2).

12. Kapil A. Taming antimicrobial resistance: a national challenge. Natl Med J India. 2015;28(1). 